\title{
EL EFECTO DE LA MATERNIDAD SOBRE LOS SALARIOS FEMENINOS EN LATINOAMÉRICA*
}

Recibido: 6 de febrero de 2014 • Aprobado 20 de agosto 2015

\author{
Héctor Alberto Botello** \\ Andrea López Alba***
}

\section{RESUMEN}

Este artículo analiza el efecto de la maternidad sobre los salarios en Latinoamérica, estudiando la tenencia del número de hijos y la estructura de las edades de los mismos. La metodología utilizada estima ecuaciones mincerianas sobre el ingreso laboral por hora con el uso adicional de la ecuación anti-sesgo de Heckman y la descomposición de Oaxaca Blinder. Los resultados muestran que las madres ganan en promedio $13 \%$ menos que las no madres; este diferencial se amplía hasta el 21 \% cuando la madre tiene niños menores de cinco años. Por cada niño adicional, el costo de oportunidad por maternidad aumenta un 1,2 \% del salario. La discriminación por parte de los empleadores y la informalidad laboral son las razones principales que explican este fenómeno.

\section{PALABRAS CLAVE}

Maternidad, brecha salarial, sesgo de selección, América Latina.

\section{CLASIFICACIÓN JEL}

J13, J16, J24, C24.

\section{CONTENIDO}

Introducción, 1. Revisión de la literatura, 2. Metodología, 3. Resultados y análisis, 4. Conclusiones, Bibliografía.

\footnotetext{
El presente artículo de investigación surge del interés personal de los autores. Los investigadores pertenecen al grupo de Investigación sobre Desarrollo Regional y Ordenamiento Territorial reconocido por Colciencias.

** Economista de la Universidad Industrial de Santander, Bucaramanga, Colombia. Profesor de la cátedra de Política Monetaria y Fiscal, Universidad Industrial de Santander, Bucaramanga, Colombia. Dirección postal: Urbanización Prados de Fátima Casa L2. Celular: 3012829726. Correo electrónico: hectoralbertobotello@gmail.com.

*** Estudiante de Economía, Universidad Industrial de Santander, Bucaramanga, Colombia. Dirección postal: Urbanización Prados de Fátima Casa L2. Celular: 3197577025. Correo electrónico: andrealopezalba@ gmail.com.
} 


\section{EFFECT OF MOTHERHOOD ON FEMALE SALARIES IN LATINAMERICA ABSTRACT}

In this article the effect of motherhood on salaries in Latin America is analyzed, studying the number of children and the structure of their ages. The methodology used estimated Mincer equations of labor income per hour with additional use of anti-bias Heckman and Blinder Oaxaca decomposition equation. Results show that mothers earn $13 \%$ less salary than women who are not mothers; this difference amounts to $21 \%$ when the mother has children younger than five years. For each additional child, the costopportunity for motherhood increases $1.2 \%$ of a woman's salary. Discrimination by employers ands labor informality are the main reasons which explain this phenomenon.

\section{KEY WORDS}

Motherhood, Salary gap, Selection bias, Latin America.

\section{JEL CLASSIFICATION}

J13, J16, J24, C24.

\section{CONTENT}

Introduction 1. Literature review 2. Proposed model Methodology 3. Results and analysis 4. Conclusions. Bibliography.

\section{O EFEITO DA MATERNIDADE SOBRE OS SALÁRIOS FEMININOS NA AMÉRICA LATINA}

\section{RESUMO}

O presente artigo examina o efeito da maternidade sobre os salários na América Latina, estudando o mandato do número de crianças e a estrutura de idades do mesmo. A metodologia utilizada equações mincerianas estimado sobre o rendimento do trabalho por hora com o uso adicional de a equação anti-sesgo de Heckman e decomposição de Oaxaca Blinder. Os resultados mostram que as mães ganham em média $13 \%$ menos do que aquelas que não são mães, esse diferencial é estendida até $21 \%$ quando a mãe tem as crianças com menos de cinco anos de idade. Para cada criança adicional, o custo de oportunidade de licença de maternidade aumenta para 1,2 \% do seu salário. À discriminação por parte dos empregadores e o trabalho informal são as principais razões que explicam este fenómeno.

\section{PALAVRAS-CHAVE}

Maternidade, diferenças salariais, o viés de seleção, na América Latina.

\section{CLASSIFICAÇÃO JEL}

J13, J16, J24, C24.

\section{CONTEÚDO}

Introdução 1. Revisão da literatura 2. Metodologia modelo proposto, 3. Resultados e análise, 4. Conclusões, Referências. 


\section{INTRODUCCIÓN}

Según las estadísticas de la Comisión Económica para América Latina (CEPAL), en el 2010 el jefe de hogar en el 30,8 \% de los hogares de la región era mujer y principal aportante de los ingresos. Esta posición conlleva una carga adicional para este género ya que diversos estudios han encontrado que existe una penalización salarial significativa asociada a la discriminación de género (Atucha y Pasquale, 2003, p. 16; Martínez y Acevedo. 2004, p. 3; Fuentes, Palma y Montero, 2005, p. 5; Peñas, 2002, p. 9).

Una de las causas citadas para explicar la desigualdad de género en el mercado laboral es la penalización salarial por maternidad; es decir, por el hecho de ser madre, la mujer incurre en una pérdida de su ingreso laboral. Lo anterior se explica porque como la mayor parte de la crianza de los hijos recae en las mujeres, estas deben realizar un trade-off entre el tiempo dedicado a su empleo y a su familia, perdiendo capacidades laborales tales como la educación, la experiencia y el know how (BenPorath, 1967, p. 4; Budig y England, 2001, p. 3; Molina y Montuenga, 2008, p. 3). Por lo anterior, las mujeres que tienen hijos y participan en el mercado laboral tienden a ser menos productivas ya que optan por emplearse en trabajos que les permitan tener horarios flexibles que se caracterizan por ofrecer menores remuneraciones (Rosen, 1986, p. 7).

De acuerdo con Hyclak (2012, p. 384), incluso, si se considerara a las madres y a las no madres en la misma ocupación y la misma productividad, una penalización salarial surgiría si los empleadores creen que el cuidado de niños y las tareas domésticas afectarán el desempeño en la empresa, lo que hace que piensen subjetivamente que las madres son menos productivas y las gratifican con menores salarios. Estos fenómenos pueden causar una menor inversión en capital humano por parte de las madres lo que llevaría a un crecimiento salarial más lento (Mincer, 1974, p. 17); Livermore, Rodgers y Siminski, 2011, p. 19).

Frente a esta problemática los países han implementado una serie de leyes para la protección de las mujeres que afrontan la maternidad; ejemplos de estas en Latinoamérica se muestran en la tabla 1. El objetivo indirecto de estas políticas ha sido el de reducir el costo de la maternidad que tienen que asumir los empleadores de manera directa, y que es asumido por el Estado en la mayoría de países a través del sistema de seguridad social y en otros pocos casos, por el individuo. 
Tabla 1. Disposiciones nacionales esenciales para la protección de la maternidad, por país.

\begin{tabular}{|c|c|c|c|c|}
\hline País & $\begin{array}{c}\text { Duración de la } \\
\text { licencia }\end{array}$ & $\begin{array}{c}\text { Valor de la licencia como \% del } \\
\text { salario }\end{array}$ & Fuente de financiación & $\begin{array}{c}\text { Tipo de } \\
\text { financiación }\end{array}$ \\
\hline Argentina & 12 semanas & $100 \%$ & Seguridad social & Obligatoria \\
\hline Bolivia & 8 semanas & $\begin{array}{l}100 \% \text { del salario mínimo + } \\
70 \% \text { de la diferencia entre } \\
\text { el salario mínimo y los in- } \\
\text { gresos regulares }\end{array}$ & Seguridad social & Obligatoria \\
\hline Brasil & 16 semanas & $100 \%$ & Seguridad social & Obligatoria \\
\hline Chile & 18 semanas & $100 \%$ hasta cierto límite & Seguridad social & Obligatoria \\
\hline Colombia & 12 semanas & $100 \%$ & Seguridad social & Obligatoria \\
\hline Ecuador & 12 semanas & $100 \%$ & $\begin{array}{l}\text { Mixta }(75 \% \text { seguridad so- } \\
\text { cial; } 25 \% \text { empleador) }\end{array}$ & Obligatoria \\
\hline México & 12 semanas & $100 \%$ & Seguridad social & Obligatoria \\
\hline Nicaragua & 12 semanas & $100 \%$ & $\begin{array}{l}\text { Mixta (60\% seguridad social; } \\
40 \% \text { empleador) } 1\end{array}$ & Obligatoria \\
\hline Panamá & 14 semanas & $100 \%$ & Mixta 6 & \\
\hline Paraguay & 12 semanas & $50 \%$ durante 9 semanas & Seguridad social & Obligatoria \\
\hline Perú & 12 semanas & $100 \%$ & Seguridad social & Obligatoria \\
\hline Uruguay & 12 semanas & $100 \%$ & Seguridad social & Obligatoria \\
\hline Venezuela & 18 semanas & $100 \%$ & Seguridad social & Obligatoria \\
\hline
\end{tabular}

Fuente: International Labour Office (2010).

El análisis de los determinantes de las brechas salariales asociadas a la penalización salarial por maternidad resulta relevante en la formulación de políticas públicas, dado que la existencia de estos diferenciales desalienta y aumenta la probabilidad de que los hogares donde las madres son las mayores perceptoras del ingreso persistan en la pobreza. Con base en lo anterior, en el presente trabajo se desea contribuir a la literatura al analizar el efecto de la maternidad sobre los salarios en nueve países de Latinoamérica mediante una metodología estandarizada con la que se puedan comparar resultados entre países, estudiando el efecto de la tenencia del número de hijos y de la estructura de las edades de los mismos.

Las secciones están organizadas de la siguiente manera. En la segunda parte se exponen diferentes trabajos que contribuyen a la estimación de los diferenciales por maternidad en varios países, comparando la metodología empleada. En la sección 3 se detallan la metodología, y los datos a utilizar con la presentación de los resultados en la sección 4 . Finalmente en la sección 5 se exponen las conclusiones. 


\section{REVISIÓN DE LA LITERATURA}

Los primeros estudios de Mincer (1974) y Becker (1985) buscaban estimar el impacto de la maternidad sobre los salarios y sugerían que las diferencias en experiencia laboral explicaban la brecha salarial entre las madres y las no madres, debido a que las primeras se dedicaban a la crianza de sus hijos. Sin embargo, trabajos más recientes que controlan dichos factores encuentran una brecha persistente, aunque menor que la inicialmente calculada (Korenman y Neumark, 1992; Jacobsen y Levin, 1995 y Waldfogel, 1995 para Estados Unidos; Baxter, 1992 para Australia y Joshi y Newell, 1989 para el Reino Unido). Las metodologías implementadas y los resultados obtenidos por algunos autores se consignaron en la tabla 1 .

Todd (2001) estima que Reino Unido y Australia son los países con mayores pérdidas en salario para las madres con dos hijos, con diferencias de $25,5 \%$ y $12 \%$, respectivamente, seguidos por Estados Unidos y Alemania con diferencias de 10.5 \% y $10.7 \%$. Por su parte, Harkness y Waldfogel (2003) estiman que los países que presentan menos diferencias salariales son Canadá, Finlandia y Suecia, inferiores al $5 \%$. Entre los trabajos se observa que en Estados Unidos y Gran Bretaña, una proporción grande de las brechas salariales está determinada por los trabajos a medio tiempo que realizan las madres (Waldfogel, 1997; Joshi, Paci y Waldfogel, 1999).

Tabla 2. Resumen de la literatura sobre la relación entre maternidad y salarios

\begin{tabular}{|l|l|l|l|}
\hline \multicolumn{1}{|c|}{ Autores } & \multicolumn{1}{|c|}{ Datos } & \multicolumn{1}{c|}{ Método } & \multicolumn{1}{c|}{ Principales resultados } \\
\hline $\begin{array}{l}\text { Baxter } \\
\text { (1992) }\end{array}$ & Australia & $\begin{array}{l}\text { Análisis de regre- } \\
\text { sión }\end{array}$ & $\begin{array}{l}\text { Existencia de brecha salarial por } \\
\text { tenencia de hijos que prevalece aun } \\
\text { controlando por experiencia laboral }\end{array}$ \\
\hline $\begin{array}{l}\text { Becker } \\
(1985)\end{array}$ & Países occidentales & $\begin{array}{l}\text { Modelo simple } \\
\text { de división ópti- } \\
\text { ma del trabajo }\end{array}$ & $\begin{array}{l}\text { Las mujeres ganan menos que los } \\
\text { hombres debido a que emplean tiem- } \\
\text { po en cuestiones del hogar, en las que } \\
\text { el cuidado de los hijos es la variable } \\
\text { de mayor peso. }\end{array}$ \\
\hline $\begin{array}{l}\text { Budig y } \\
\text { England } \\
\text { (2001) }\end{array}$ & $\begin{array}{l}\text { Encuesta Longitudinal } \\
\text { Nacional de la Juventud } \\
\text { 1982-1993 }\end{array}$ & $\begin{array}{l}\text { Modelos de efec- } \\
\text { tos fijos }\end{array}$ & $\begin{array}{l}\text { Penalización salarial de 7 \% por hijo. } \\
\text { Controlando por experiencia laboral, } \\
\text { la penalización se reduce a 5\% }\end{array}$ \\
\hline $\begin{array}{l}\text { Harkness y y } \\
\text { Waldfogel } \\
\text { (2003) }\end{array}$ & $\begin{array}{l}\text { ta de empleo y salarios } \\
\text { (LIS) de Australia 1994, } \\
\text { Canadá 1994, Reino Uni- } \\
\text { do 1995, EE.UU 1994, } \\
\text { Alemania 1994, Finlan- } \\
\text { dia 1991 y Suecia (LNU, } \\
\text { 1991). }\end{array}$ & $\begin{array}{l}\text { Modelos de em- } \\
\text { pleo y salarios }\end{array}$ & $\begin{array}{l}\text { Controlando por sesgo de selección, } \\
\text { los países que presentan menos } \\
\text { diferencias salariales son Canadá, } \\
\text { Finlandia y Suecia, inferiores al 5 \% }\end{array}$ \\
\hline
\end{tabular}




\begin{tabular}{|c|c|c|c|}
\hline Autores & Datos & Método & Principales resultados \\
\hline $\begin{array}{l}\text { Korenman, } \\
\text { y Neumark. } \\
\text { (1992) }\end{array}$ & $\begin{array}{l}\text { Encuesta Longitudinal } \\
\text { Nacional de Mujeres Jó- } \\
\text { venes (28-38 años) } 1982\end{array}$ & $\begin{array}{l}\text { Estimaciones de } \\
\text { primera diferen- } \\
\text { cia, estimaciones } \\
\text { OLS, control de } \\
\text { sesgo de endo- } \\
\text { geneidad }\end{array}$ & $\begin{array}{l}\text { Incluyendo la experiencia laboral las } \\
\text { estimaciones por OLS atenúan pero } \\
\text { no eliminan la brecha entre salarios e } \\
\text { hijos. Variables instrumentales sugie- } \\
\text { ren que el estado civil y el número de } \\
\text { hijos son exógenas a las ecuaciones } \\
\text { de ingresos. Controlando el sesgo } \\
\text { de endogeneidad tales variables no } \\
\text { resultan exógenas }\end{array}$ \\
\hline $\begin{array}{l}\text { Molina y } \\
\text { Montuenga, } \\
\text { V. (2008) }\end{array}$ & $\begin{array}{l}\text { Panel de Hogares de la } \\
\text { Unión Europea (PHO- } \\
\text { GUE, 1994-2001). }\end{array}$ & $\begin{array}{l}\text { Efectos fijos y } \\
\text { aleatorios }\end{array}$ & $\begin{array}{l}\text { Incluyendo variables observables y no } \\
\text { observables, en España las madres } \\
\text { pierden el } 9 \% \text { de su salario normal. } \\
\text { Este valor incrementa a medida que } \\
\text { se tienen más hijos }\end{array}$ \\
\hline $\begin{array}{l}\text { Olarte y } \\
\text { Peña, (2010) }\end{array}$ & $\begin{array}{l}\text { Encuesta Nacional de } \\
\text { Calidad de Vida (2008). }\end{array}$ & $\begin{array}{l}\text { Derivaciones } \\
\text { de la ecuación } \\
\text { salarial de Min- } \\
\text { cer (1974), des- } \\
\text { com posición } \\
\text { Blinder-Oaxaca } \\
\text { y metodología de } \\
\text { Heckman (1979) }\end{array}$ & $\begin{array}{l}\text { Las madres ganan en promedio } 17.6 \% \\
\text { menos que las no madres. Luego de } \\
\text { controlar por variables observables y } \\
\text { sesgo de selección, aún persiste una } \\
\text { brecha salarial de } 9.4 \% \text { entre madres y } \\
\text { no madres; esta se hace más alta ( } 18.4 \\
\text { \%) cuando los hijos tienen menos de } \\
5 \text { años de edad }\end{array}$ \\
\hline $\begin{array}{l}\text { Piras y Ri- } \\
\text { pani (2005). }\end{array}$ & $\begin{array}{l}\text { Encuesta de Hogares } \\
1999 \text { de cada uno de los } \\
\text { países: Pesquisa Nacio- } \\
\text { nal por Amostra de Do- } \\
\text { micilios (PNAD) Brasil, } \\
\text { Encuesta de condiciones } \\
\text { de vida de Ecuador (ECV- } \\
\text { 1998), Encuesta continua } \\
\text { de Hogares para Bolivia } \\
\text { (ECH), Encuesta Nacio- } \\
\text { nal de Hogares para Perú } \\
\text { (2000) }\end{array}$ & $\begin{array}{l}\text { Función estándar } \\
\text { de ingresos para } \\
\text { corte transversal, } \\
\text { con diferentes } \\
\text { especificaciones } \\
\text { para estudiar va- } \\
\text { riados casos }\end{array}$ & $\begin{array}{l}\text { Madres con hijos menores de } 7 \text { años } \\
\text { tienen mayor participación en el } \\
\text { mercado laboral que las mujeres que } \\
\text { no tienen hijos. En Perú las madres } \\
\text { muestran penalizaciones laborales de } \\
10,7 \% \text { por hora, mientras que en Boli- } \\
\text { via y Brasil reciben una prima laboral, } \\
\text { de } 19,3 \% \text { y } 6,8 \% \text {, respectivamente. } \\
\text { En Ecuador no se notan efectos } \\
\text { significativos }\end{array}$ \\
\hline
\end{tabular}

Fuente: elaboración propia

Para América Latina, Piras y Ripani (2005) estiman la brecha familiar para Bolivia, Brasil, Ecuador y Perú, sin corregir el sesgo de selección. Los resultados sugieren que en Perú existe una penalización salarial de 11 \%, aproximadamente, por la presencia de un hijo, mientras que en Brasil, por el contrario, existe una ganancia de 8,1 \% por la presencia de dos o más hijos. En cuanto a Ecuador y Bolivia, las autoras encuentran que la brecha familiar no es estadísticamente significativa. Concluyen entonces que los resultados para Latinoamérica no muestran un claro impacto de la maternidad sobre los salarios a diferencia de las muestras en los países desarrollados. 
Para analizar el caso en Colombia, Gutiérrez (2008) utiliza los datos de la Encuesta Nacional de Calidad de Vida 2003 involucrando los salarios del sector urbano y del rural. Sus resultados sugieren que la brecha familiar es de un $51 \%$; al corregir por sesgo de selección esta aumenta a 65,9\%. Los instrumentos elegidos se encuentran correlacionados con otras variables de interés como la educación propia, y por ende los resultados tan extremos pueden ser producto de este sesgo. En contraposición, Olarte y Peña (2010) encontraron evidencia de que ser madre impacta de manera positiva la probabilidad de trabajar en lugares de baja calidad como en un puesto ambulante; en contraposición, experimentan una menor probabilidad de tener trabajos en los cuales contribuyan a una pensión y a salud. Concluyen que controlando por estado civil y otras variables dependientes, al ser madre existe mayor posibilidad de ocuparse en oficios del hogar, pese a trabajar siendo este efecto más notorio en las madres con hijos entre 0 y 5 años. Las autoras calculan que la brecha salarial bruta por maternidad es de alrededor de $17.6 \%$ para mujeres entre 18 y 65 años de edad. Corrigiendo el sesgo de selección y controlando factores observables como el capital humano, la estructura del hogar y la región, aún subyace un diferencial del $9.4 \%$, el cual se hace más pronunciado para mujeres con hijos en edades entre los 0 y los 5 años de edad.

Sin embargo, hay que mencionar que Livermore, Rodgers y Siminski (2011, p. 12) afirman que la evidencia de lo anterior puede ser circunstancial ya que otros autores no han encontrado penalización salarial por maternidad. Esto se da especialmente en países donde las mujeres cuentan con altos niveles de educación y una red de seguridad social lo suficientemente permisiva para que la jornada laboral no interrumpa con sus labores hogareñas. Ejemplos de lo anterior son los estudios en Dinamarca de Gupta y Smith (2002) y en Suecia por parte de Albrecht, Sundström y Vroman (1999).

Con base en la literatura investigada, se busca estimar las brechas por maternidad en nueve países de Latinoamérica controlando factores observables de las madres y no madres. Con este objetivo se exponen a continuación el modelo y la fuente de datos utilizados para la medición de las brechas salariales por maternidad.

\section{METODOLOGÍA}

\subsection{Modelo de Mincer}

El primer modelo utilizado para estimar las brechas salariales entre madres y no madres es el modelo de Mincer (1974); con este se estima los efectos posibles que puede tener cada una de las características de los trabajadores sobre sus ingresos 
laborales (Psacharopoulos y Patrinos 2004; Cohn y Addison, 1998; Asplund y Pereira, 1999, Harmon y otros, 2001, 2003).

La ecuación de Mincer (1974)se calcula sobre un modelo semilogarítmico por mínimos cuadrados ordinarios (MCO), teniendo como variable dependiente el logaritmo de los ingresos laborales y como variables independientes características socioeconómicas del trabajador (ecuación 1). Generalmente los datos implementados en estos modelos provienen de las encuestas de empleo de cada país que son observaciones de corte transversal.

$$
\operatorname{Ln}(\mathrm{Y})=\beta_{0}+\beta_{1} \mathrm{~S}+\beta_{2} \operatorname{Exp}+\beta_{3} \operatorname{Exp}^{2}+\beta_{4} \mathrm{~L}+\varepsilon
$$

Donde:

- Y son los ingresos laborales del individuo

- $\quad$ S es el número de años de educación formal completada

- Exp son los años de experiencia laboral

- L corresponde a otras variables del trabajador.

- $\varepsilon$ es el término de perturbación aleatoria que se distribuye según una distribución normal.

Los coeficientes $(\beta)$ se interpretan como el aumento o la disminución porcentual sobre el ingreso laboral por hora generado cada una de las características de los trabajadores. Una vez se ha hecho el modelo de regresión lineal, se procede a realizar las pruebas de calibración de los modelos tal como son la validación de los supuestos de normalidad de las variables, la homocedasticidad, la no colinealidad y la no omisión de variables del modelo; en caso de no cumplirse alguno de los supuestos anteriores se procederá a la re-estimación. La efectividad de este modelo se mide a través de la varianza de la variable dependiente captada por parte de las variables de control, este indicador se denomina $\mathrm{R}^{2}$, si este es más alto; más efectivo es el modelo en determinar el comportamiento de la variable dependiente.

Pero existe un problema implícito en los datos, denominado sesgo de selección. Este consiste principalmente en la ausencia de aleatoriedad o selección muestral no aleatoria dentro de las encuestas de hogares que se realizan (Esquivel, 2007 y Rivera, 2013). Se define como sesgo porque una muestra que está concentrada en cierto grupo poblacional desvía el resultado obtenido de una variable de interés hacia el grupo que tenga mayor representación. Este problema es especialmente grave cuando se investiga sobre diferencias en los ingresos laborales de las personas, 
no permitiendo obtener resultados exactos del fenómeno analizado, dado que la encuesta no toma en cuenta la probabilidad de participar en el mercado laboral.

La corrección de este problema se puede llevar a cabo de diferentes formas (Rivera, 2013); la más utilizada es la corrección de Heckman y se procede estimando dos regresiones. La primera estimación se efectúa a base de la ecuación de participación, entendida como la probabilidad de que el individuo participe en el mercado laboral contra los factores que pueden incidir en la elección (Heckman, 1979).

$$
p i=\beta 0+z i \varphi+u i
$$

Donde pi se refiere a la probabilidad de participar en el mercado laboral; zi corresponde a un vector con variables explicativas que influyen en la decisión de participar; $\phi$ es un vector de parámetros, y ui son los errores. Después de aplicada, el segundo cálculo es la ecuación de Mincer incorporando la corrección de selección realizada por lambda $(\lambda)$ como inversa del ratio de Mill (Rivera, 2013)

$$
\log (Y)=\beta_{0}+\beta_{1} S+\beta_{2} \operatorname{Exp}+\beta_{3} \operatorname{Exp}^{2}+\beta_{4} L+\lambda t i \theta+\varepsilon i
$$

Con ello se ha corregido el sesgo de selección. Usando el argumento de Torres y Celton (2009), la importancia del uso del $(\lambda)$ se determina al estimar la regresión, siempre y cuando su coeficiente sea significativo. En el caso de no ser representativo, se podría trabajar directamente sin la necesidad de la corrección. Para este trabajo se obtuvo mediante la maximización de la función de verosimilitud.

Con estas consideraciones, el resultado final de estos modelos es la estimación del efecto neto que tiene sobre el ingreso laboral por hora el ser madre o no. Sin embargo, Olarte y Peña (2010) sugieren que existen diferentes formas de analizar el efecto de la maternidad sobre los salarios cambiando la naturaleza de la variable independiente que aproxima empíricamente tal fenómeno (la maternidad). En este sentido, el presente estudio explora cuatro tipos de efectos. En la primera especificación se valora únicamente si la madre tiene hijos o no. En la segunda se divide entre hijos de menos de 5 años y más de cinco años. En la tercera se introduce una variable única continua que mide el número de niños dentro del hogar, y en la cuarta especificación se observa el efecto de tener uno, dos o más de dos hijos.

Sin embargo, lo anterior no permite conocer el origen del fenómeno, es decir, si las brechas en los salarios de las madres y no madres provienen de sus dotaciones socioeconómicas o por el hecho mismo de la maternidad. Lo anterior se comprende con la ayuda de la descomposición Oaxaca Blinder la cual se explica a continuación. 


\subsection{Descomposición de Oaxaca Blinder}

En este modelo, se debe escoger una variable continua como dependiente (Y) y una serie de predictores $(\mathrm{X})$ que son propios de dos grupos de análisis: uno de control y otro experimental. Para esta aplicación específica, el logaritmo de los salarios es la variable a estudiar, el vector de variables predictores son las características socioeconómicas de los individuos, y los grupos de análisis se organizan entre mujeres que tienen hijos y mujeres que no los tienen. Lo que se estima en este modelo es la diferencia entre ingresos, dada la condición de separación teniendo en cuenta cada una de las características de los trabajadores; así se sabrá de dónde proviene la brecha entre ingresos.

Econométricamente las ecuaciones a calcular son:

$$
Y_{i}=X_{i}^{\prime} \beta_{i}+u_{i}\left|E\left(u_{i}=0\right)\right| i \text { ò } A, B
$$

Siendo A y B los grupos a controlar y u el término de error de la estimación. Se espera encontrar la diferencia dentro del valor esperado de los salarios para cada grupo.

$$
\mathrm{R}=\mathrm{E}\left(\mathrm{Y}_{\mathrm{A}}\right)-\mathrm{E}\left(\mathrm{Y}_{\mathrm{B}}\right)
$$

Si se reemplaza 4 en 5 y se asume $\mathrm{E}\left(u_{i}=0\right)$, se tiene que:

$$
R=E\left(X_{A}\right)^{\prime} \beta_{A}-E\left(X_{B}\right)^{\prime} \beta_{B}
$$

Para entender todos los efectos que se pueden extraer de la ecuación anterior, se puede realizar la descomposición algebraica expuesta por Daymont y Andrisani $(1984)^{1}$ :

$\mathrm{R}=\left[\mathrm{E}\left(\mathrm{X}_{\mathrm{A}}\right)-\mathrm{E}\left(\mathrm{X}_{\mathrm{B}}\right)\right]^{\prime} \beta_{\mathrm{A}}+\left[\mathrm{E}\left(\mathrm{X}_{\mathrm{A}}\right)^{\prime}\left(\beta_{\mathrm{A}}-\beta_{\mathrm{B}}\right)\right]+\left[\mathrm{E}\left(\mathrm{X}_{\mathrm{A}}\right)-\mathrm{E}\left(\mathrm{X}_{\mathrm{B}}\right)\right]^{\prime}\left(\beta_{\mathrm{A}}-\beta_{\mathrm{B}}\right)$

Estos tres términos conforman los efectos de las interacciones posibles entre los coeficientes, las variables y los valores esperados del ingreso. La ecuación siete se puede resumir como una ecuacion que representa una descomposición three-fold. El primer término es la parte del diferencial atribuible a los efectos de los predictores, tales como la educación y la experiencia del individuo y las características de la firma donde trabaja. El segundo mide las referidas a los coeficientes (incluyendo el intercepto) según los grupos de control; en general estos son los considerados como

Para mayor ampliación de la explicación matemática, se puede revisar el artículo citado en el pie de página 6 del documento de Daymont y Andrisani (1984). 
los originados por la característica de control, que para este caso es la brecha salarial por maternidad. El último opera sobre las interacciones entre los dos términos antes mencionados. Es importante señalar que para efectos de estimación econométrica los ingresos laborales esperados, calculados a partir de la descomposición de Oaxaca Blinder, han sido estimados con base en la ecuación minceriana con sesgo de corrección de Heckman de la ecuación (3).

El procedimiento de la estimacion es por medio de minimos cuadrados ordinarios - $\mathrm{MCO}-$ a través de una funcion semilogarítmica, obteniendo por separado los valores esperados de los ingresos laborales para ambos grupos, junto con sus errores estándar, tal y como lo haría una regresión de Mincer. Además, se calcula el valor esperado si cada uno de los grupos tuviera las caracteristicas del otro.

En este punto hay que mencionar que existe otro tipo de descomposición, la cual parte de la existencia de un conjunto de coeficientes que no son causantes de las brechas salariales por maternidad, pero al mismo tiempo son claves para encontrar las diferencias en los predictores. Supóngase que es un coeficiente hipotético que se puede reemplazar en la ecuación (7) como sigue:

$$
R=\left[E\left(X_{A}\right)-E\left(X_{B}\right)\right]^{\prime} \beta^{*}+\left[E\left(X_{A}\right)^{\prime}\left(\beta_{A}-\beta^{*}\right)\right]+\left[E\left(X_{A}\right)-E\left(X_{B}\right)\right]^{\prime}\left(\beta_{A}-\beta^{*}\right)
$$

A partir de la ecuación (8) se pueden extraer dos efectos: el primero es la parte de la desigualdad que es explicada por las diferencias en los predictores. El segundo efecto es la unión de los dos últimos términos:

$$
U=\left[E\left(X_{A}\right)^{\prime}\left(\beta_{A}-\beta^{*}\right)\right]+\left[E\left(X_{A}\right)-E\left(X_{B}\right)\right]^{\prime}\left(\beta_{A}-\beta^{*}\right)
$$

La anterior ecuación se refiere a la parte no explicada por los predictores, la cual se le atribuye, frecuentemente, a la tenencia de hijos. No obstante, esta tambien captura el efecto de variables no especificadas dentro del modelo. El procedimiento para esta segunda descomposición es más complejo, aunque más robusto, dado que el cálculo sobre el beta hipotético debe hacerse. Una suposicion a considerar es planteada por Oaxaca (1973), quien toma $\beta_{A}=\beta^{*}$ y $\beta_{B}=\beta^{*}$. Es decir, que los grupos se intercalan: el grupo A que correspondería a las mujeres que no tienen hijos, y el B sería de mujeres que sí los tienen. Otra suposición, que también procede de Oaxaca (1973), es pensar de que existe discriminación de una vía, es decir, únicamente las mujeres con hijos se presentan como discriminadas. Sobre la ecuación (9):

$$
\mathrm{R}=\left[\overline{\mathrm{X}_{\mathrm{A}}}-\overline{\mathrm{X}_{\mathrm{B}}}\right] \cdot \hat{\beta}_{\mathrm{A}}+\mathrm{X}_{\mathrm{B}}\left(\hat{\beta}_{\mathrm{A}}-\hat{\beta}_{\mathrm{B}}\right)
$$


Pero no hay razón para suponer inicialmente que hay discriminación hacia algún grupo en particular. Por esto se tiende a configurar el $\beta^{*}$ como una suma ponderada (Cotton, 1988; Reimers, 1983); con este cálculo se pueden corregir los sesgos de selección dentro del mercado laboral para las madres. Ejemplos de ecuaciones que representan la anterior suposición sobre la descomposición son:

$$
\begin{aligned}
& \beta^{*}=\frac{n_{\mathrm{A}}}{n_{\mathrm{A}}+n_{\mathrm{B}}} \hat{\beta}_{\mathrm{A}}+\frac{n_{\mathrm{B}}}{n_{\mathrm{A}}+n_{\mathrm{B}}} \hat{\beta}_{\mathrm{B}} \\
& \beta^{*}=0.5 \hat{\beta}_{\mathrm{A}}+0.5 \hat{\beta}_{\mathrm{B}}
\end{aligned}
$$

Observando las diferentes metodologías, para este trabajo se utilizaron las ponderaciones dadas por el tamaño originario de los grupos, mostradas en la ecuación (12). Para el cálculo de las estimaciones econométricas se utilizó el programa Stata en su versión 12.

\subsection{Fuentes de datos}

Los datos utilizados se obtuvieron de la Encuesta para estudios sociales de la Corporación Andina de Fomento (CAF) del año 2012. Esta encuesta contiene unos módulos generales que se aplican todos los años y ofrecen mediciones de acceso, calidad, gasto y satisfacción con una serie de servicios básicos en las áreas de transporte urbano, seguridad, recolección de basura, agua y saneamiento, energía eléctrica y vivienda ${ }^{2}$. El beneficio de usar esta encuesta para investigar tendencias del mercado laboral latinoamericano es la homogeneización de las preguntas en relación con las características del trabajo. La encuesta de 2012 se aplicó entre el 21 de agosto y 19 de octubre en nueve países: Argentina, Bolivia, Brasil, Colombia, Ecuador, Perú, Uruguay, Venezuela y Panamá. La población total de la encuesta fue de 9 mil adultos entre 25 y 65 años de edad con un rango total ajustado con factores de expansión de 39 millones de personas, distribuidas de la siguiente manera:

Tabla 3. Distribución de los encuestados por país

\begin{tabular}{|l|c|c|c|c|}
\hline \multicolumn{1}{|c|}{ País } & Hombres & Mujeres & $\begin{array}{c}\text { Mujeres que } \\
\text { trabajan }\end{array}$ & \% Madres \\
\hline Argentina & 1.433 .960 & $1,709.602$ & 795.683 & $82,70 \%$ \\
\hline Bolivia & $1.172,128$ & 1.182 .430 & 705.106 & $91,36 \%$ \\
\hline
\end{tabular}

La información del cuestionario y la metodología ampliada del muestreo de la encuesta se puede observar en CAF (2012). 
El efecto de la maternidad sobre los salarios femeninos en Latinoamérica

\begin{tabular}{|l|r|r|r|r|}
\hline Brasil & 7.663 .775 & $8,721.426$ & 4.755 .416 & $83,33 \%$ \\
\hline Colombia & 2.341 .238 & 2.700 .690 & 1.455 .207 & $87,77 \%$ \\
\hline Ecuador & 842.831 & 880.854 & 430.735 & $90,52 \%$ \\
\hline Perú & 2.415 .774 & $2.579,855$ & 1.214 .719 & $86,65 \%$ \\
\hline Uruguay & 364,323 & 415.063 & 252,078 & $75,22 \%$ \\
\hline Venezuela & $1,182.152$ & 1.302 .058 & 743,982 & $81,58 \%$ \\
\hline Panamá & 142.784 & 180.232 & 103.604 & $79,79 \%$ \\
\hline Total & 17.558 .964 & 19.672 .211 & 10.456 .529 & $84,77 \%$ \\
\hline
\end{tabular}

Fuente: elaboración propia con base en encuesta para estudios sociales de la CAF (2012)

De estos datos se puede estimar que la tasa de ocupación laboral femenina es de $53 \%$ con un 84,77 \% con hijos. Bolivia y Uruguay son los países con mayores tasas de participación femenina con el $60 \%$, mientras que las tasas de maternidad sobrepasan el 85 \% en Bolivia y Ecuador.

En la tabla 4 se muestran las estadísticas descriptivas de las mujeres ocupadas separadas entre las que tienen hijos contra los que no tienen hijos de la base de datos de la CAF. Las madres tienen una media de edad 5 años más alta con el mismo grado de dispersión; igualmente existe un alto porcentaje de madres solteras trabajadoras, $41 \%$ no viven con su pareja.

Tabla 4. Análisis descriptivo de los hogares de las mujeres que tienen y no tienen hijos y que participan en el mercado laboral

\begin{tabular}{|l|l|c|c|}
\hline \multicolumn{1}{|c|}{ Variable } & \multicolumn{1}{|c|}{ Característica } & No Tienen & Tienen \\
\hline \multirow{2}{*}{ Edad } & Media & 35,70 & 40,75 \\
\cline { 2 - 4 } & Desviación & $(10.83)$ & $(10)$ \\
\hline \multirow{4}{*}{ Vive con la pareja } & Sí & $28,29 \%$ & $59 \%$ \\
\hline \multirow{4}{*}{$\begin{array}{l}\text { Máximo nivel educati- } \\
\text { vo alcanzado }\end{array}$} & Ninguno & $1 \%$ & $1 \%$ \\
\cline { 2 - 4 } & Primaria & $7 \%$ & $30 \%$ \\
\cline { 2 - 4 } & Secundaria & $37 \%$ & $45 \%$ \\
\cline { 2 - 4 } & Técnica & $16 \%$ & $11 \%$ \\
\cline { 2 - 4 } & Universitaria & $40 \%$ & $13 \%$ \\
\hline
\end{tabular}


Héctor Alberto Botello - Andrea López Alba

\begin{tabular}{|c|c|c|c|}
\hline \multirow{7}{*}{$\begin{array}{l}\text { Rangos del ingreso } \\
\text { mensuales del hogar } \\
\text { en dólares de } 2012\end{array}$} & Menos de $\$ 100$ & $0 \%$ & $1 \%$ \\
\hline & De \$101 a \$200 & $1 \%$ & $3 \%$ \\
\hline & De \$201 a \$400 & $7 \%$ & $16 \%$ \\
\hline & De $\$ 401$ a $\$ 800$ & $28 \%$ & $38 \%$ \\
\hline & De \$801 a \$1.600 & $29 \%$ & $31 \%$ \\
\hline & De $\$ 1.601$ a $\$ 3.200$ & $20 \%$ & $9 \%$ \\
\hline & Más de $\$ 3.200$ & $16 \%$ & $3 \%$ \\
\hline \multirow{3}{*}{ Tipo de trabajador } & Cuenta propia & $18.7 \%$ & $36.1 \%$ \\
\hline & Patrona o empleadora & $2.8 \%$ & $6.1 \%$ \\
\hline & Obrera o empleada & $78,5 \%$ & $57,8 \%$ \\
\hline \multirow{2}{*}{ Jornada laboral } & Completa & $80,7 \%$ & $84,6 \%$ \\
\hline & Parcial & $19,3 \%$ & $15,4 \%$ \\
\hline $\begin{array}{l}\text { Tenencia seguridad } \\
\text { social }\end{array}$ & Sí & $67,3 \%$ & $47,4 \%$ \\
\hline \multirow{8}{*}{ Tamaño de la empresa } & Una persona & $20,4 \%$ & $39,0 \%$ \\
\hline & De 2 a 3 personas & $11,3 \%$ & $15,1 \%$ \\
\hline & De 4 a 5 personas & $6,0 \%$ & $7,7 \%$ \\
\hline & De 6 a 10 personas & $11,5 \%$ & $11,1 \%$ \\
\hline & De 11 a 20 personas & $10,0 \%$ & $6,5 \%$ \\
\hline & De 21 a 50 personas & $14,7 \%$ & $7,8 \%$ \\
\hline & De 51 a 100 personas & $5,8 \%$ & $3,9 \%$ \\
\hline & Más de 100 personas & $20,4 \%$ & $8,9 \%$ \\
\hline \multirow{3}{*}{ Lugar de trabajo } & Vivienda propia o ajena & $19,7 \%$ & $34,6 \%$ \\
\hline & Local de la empresa & $71,8 \%$ & $51,9 \%$ \\
\hline & ambulante & $8,5 \%$ & $13,5 \%$ \\
\hline \multirow{7}{*}{$\begin{array}{l}\text { Rangos ingreso laboral } \\
\text { en dólares de } 2012\end{array}$} & Menos de $\$ 100$ & $0,9 \%$ & $6,2 \%$ \\
\hline & De \$101 a \$200 & $6,6 \%$ & $9,9 \%$ \\
\hline & De \$201 a \$400 & $29,2 \%$ & $39,2 \%$ \\
\hline & De $\$ 401$ a $\$ 800$ & $33,6 \%$ & $30,8 \%$ \\
\hline & De $\$ 801$ a $\$ 1600$ & $13,3 \%$ & $9,7 \%$ \\
\hline & De \$1601 a \$3200 & $8,3 \%$ & $2,8 \%$ \\
\hline & Más de \$3200 & $8,0 \%$ & $1,4 \%$ \\
\hline
\end{tabular}

Fuente: encuesta para estudios sociales de la CAF (2012) 
En relación con las dotaciones en capital humano, se observa que un $13 \%$ de las mujeres que tienen hijos cursaron grado universitario, contra el $40 \%$ de las que no tienen hijos; igualmente, mientras que el $30 \%$ de las madres tienen primaria, solo un $7 \%$ de las no madres poseen este mismo grado. Cuando se distribuyen los hogares por rangos de ingresos en dólares del 2012, se calcula que las madres viven en hogares donde el ingreso total mensual se ubica en los rangos más bajos. Por ejemplo solo un $3 \%$ de las madres tienen ingresos por encima de los 3.200 dólares contra el $16 \%$ de las que no lo son.

En resumen, se observa que las madres ocupadas poseen niveles de educación más bajos, en comparación con las que no son madres, y a primera vista esta característica se ve reflejada en sus ingresos mensuales. No obstante, es necesaria una revisión de las características laborales para especificar otras fuentes de esta afirmación.

\subsection{Análisis laboral}

Las madres de los países encuestados suelen ser relativamente más independientes que su contraparte. Por ejemplo, el $36 \%$ de las madres son trabajadoras por cuenta propia contra el $18,7 \%$ de las no madres, mientras que un $6,1 \%$ son patronas contra el 2,8 \% de las que no lo son. En este sentido, los grupos comparados operan sobre una jornada laboral similar con el $84 \%$ de las mujeres que tienen hijos y el $80 \%$ de las que no tienen laboran en jornada completa. No obstante, no poseen los mismos niveles de seguridad social ya que solo el $47 \%$ de las madres poseen este beneficio mientras que el $67 \%$ de las no madres lo poseen.

Más del $75 \%$ de las mujeres ocupadas que tienen hijos desempeñan labores en empresas de menos de 10 personas contra el $49 \%$ de las que no tienen hijos; en cambio, en las empresas de más de 51 personas solo labora un $13 \%$ de las madres contra un $26 \%$ de las no madres. En términos de ubicación, un $35 \%$ de las mujeres que tienen hijos laboran en empresas informales dentro de la vivienda propia o ajena, frente al 19,7 \% de las no madres; además, el 13,5\% de las madres tienen puestos ambulantes y solo un 52 \% laboran en locales fijos de las empresas.

De este análisis descriptivo se puede determinar que el mercado laboral de las madres es mayoritariamente informal caracterizado por su baja productividad (Rodríguez e Higa, 2009; Samaniego, 2009; Cárdenas y Rozo, 2009) que limita sus ingresos laborales y puede causar los diferenciales en la obtención de ingresos laborales. Se espera profundizar y descomponer las causas de este fenómeno con la utilización de modelos econométricos expuestos anteriormente. 


\section{RESULTADOS Y ANÁLISIS}

En la tabla 6 se muestran los resultados de las estimaciones de modelo de Mincer especificados en la sección anterior sobre la ecuación 1, utilizando las variables disponibles para las mujeres ocupadas dentro de la encuesta de servicios sociales de la CAF en el año 2012. Se muestran las observaciones totales, el $R^{2}$ y las variables tanto continuas como categóricas y el efecto porcentual que tienen sobre el ingreso laboral promedio.

Después de depurar la base de datos y extraer las observaciones perdidas y dispersas, se analizaron los salarios de cerca de 5,7 millones de mujeres trabajadoras. El porcentaje de varianza estimado por el modelo de Mincer es cercano al 52 \%, siendo el mejor modelo la especificación 4 referida al análisis de tenencia de niños por número. En relación con su interpretación, el efecto de las variables continuas se interpreta como el cambio porcentual que tendrá la variable dependiente con una variación del $1 \%$ de la variable independiente; por ejemplo, un aumento del $1 \%$ en la edad de la trabajadora, incrementa en un $0,0174 \%$ el ingreso laboral promedio. Por otro lado, en las variables categóricas, el cambio porcentual sobre el puntaje se da con relación a una característica base. Por ejemplo, la tenencia de un grado de maestría o doctorado incrementa entre un 66 \% y 72 \% el salario en comparación con las mujeres que no han cursado ningún grado escolar.

Sobre las variables de interés para la tesis central de este trabajo, la tenencia de hijos es el origen de brechas salariales importantes dentro del grupo de mujeres ocupadas. La penalización por maternidad es en promedio un 13 \% (especificación 1); no obstante, cuando se interactúa este efecto con la variable de nivel educativo se observa que la brecha no afecta a las mujeres con grados educativos menores a niveles secundarios. De hecho, las mujeres que tienen hijos y no han cursado ningún grado educativo ganan $13 \%$ más que las mujeres que no tienen hijos, igual sucede con el grado de primaria (20\%) y secundaria (1\%). Sin embargo, en los grados de técnica, universitaria y posgrado la brecha es negativa y las madres ganan un 55\% 14 \% y $35 \%$ menos que las no madres; estos últimos tramos de la distribución causan que el diferencial total sea del $13 \%$. Esta tendencia sería contraria al argumento de Livermore, Rodgers y Siminski (2011) que justifica la ausencia de penalizaciones por maternidad en países donde los niveles de educación son altos y hay amplias leyes de seguridad social. Sin embargo, la alta informalidad y la discriminación empresarial siguen siendo factores institucionales y culturales que afectan de manera significativa el mercado laboral latinoamericano. 
El efecto de la maternidad sobre los salarios femeninos en Latinoamérica

Tabla 5. Salarios estimados (en logaritmo) y diferencial salarial entre mujeres que tienen hijos y no tienen hijos por nivel educativo

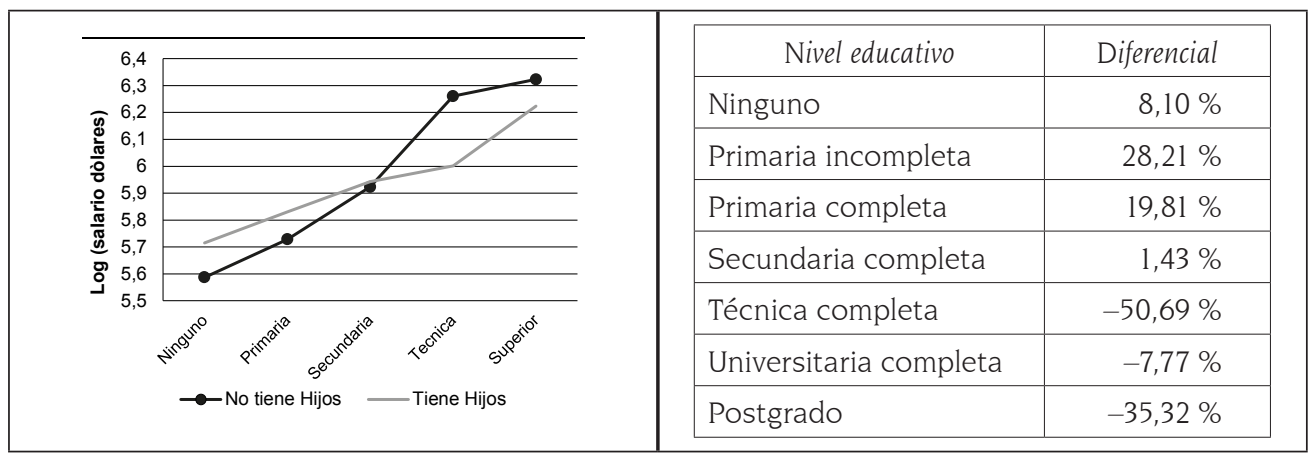

Fuente: cálculos de los autores. Encuesta para estudios sociales de la CAF (2012)

La brecha por maternidad cuando se tienen niños mayores de cinco años llegar a ser hasta el 21 \% (especificación 2), mientras que en las mujeres que tienen niños de más de cinco años la brecha es del 6 \%. Lo anterior apoya la creencia de que los infantes más pequeños requieren muchos más cuidados y atenciones de sus madres, haciéndoles tener una desventaja mayor en el mercado laboral. En términos comparativos, la región se coloca dentro del grupo del Reino Unido, Australia, Alemania y Estados Unidos para los cuales la penalización salarial para dos hijos es de $25.5 \%$, 12 \%, 10.7 \% y $10.5 \%$, respectivamente (Todd, 2001), y bastante lejos de países como Canadá, Finlandia y Suecia, para los cuales la penalización salarial correspondiente a dos hijos se encuentra en valores inferiores al 5 \% (Harkness y Waldfogel, 2003).

Tabla 6. Resultados de las estimaciones. Variable dependiente: ingreso laboral por hora Mínimos cuadrados ordinarios con corrección de Heckman

\begin{tabular}{|c|c|c|c|c|c|c|}
\hline \multirow{2}{*}{ Variable } & \multirow{2}{*}{ Característica } & \multicolumn{4}{|c|}{ Especificación del Modelo } & \multirow{2}{*}{$\begin{array}{c}\text { Característica } \\
\text { Base }\end{array}$} \\
\hline & & (1) & (2) & (3) & (4) & \\
\hline \multirow{2}{*}{ Edad } & Edad & $0,0174(0,04)$ & $0,0073(0,04)$ & $0,0129(0,07)$ & $0,0144(0,07)$ & \\
\hline & $\operatorname{Edad}^{\wedge} 2$ & $-0,0001(0,00)$ & $-0,001(0,00)$ & $-0,0001(0,00)$ & $-0,0001(0,00)$ & \\
\hline $\begin{array}{l}\text { Horas de } \\
\text { Trabajo }\end{array}$ & Continua & $0,0022(0,05)$ & $0,0023(0,07)$ & $0,0022(0,06)$ & $0,0021(0,03)$ & \\
\hline Origen & Nació en este país & $0,1044(0,05)$ & $0,09(0,02)$ & $0,1557(0,01)$ & $0,0726(0,03)$ & En otro país \\
\hline \multirow{3}{*}{$\begin{array}{l}\text { Situación } \\
\text { Laboral }\end{array}$} & Patrón & $0,4686(0,04)$ & $0,51(0,02)$ & $0,4743(0,04)$ & $0,4722(0,07)$ & $\begin{array}{l}\text { Cuenta } \\
\text { Propia }\end{array}$ \\
\hline & $\begin{array}{l}\text { Empleado jornada } \\
\text { completa }\end{array}$ & $-0,0311(0,06)$ & $-0,01(0,03)$ & $-0,0326(0,09)$ & $-0,0045(0,06)$ & \\
\hline & $\begin{array}{l}\text { Empleado a tiempo } \\
\text { parcial }\end{array}$ & $-0,1279(0,05)$ & $-0,1097(0,05)$ & $-0,1365(0,09)$ & $-0,101(0,04)$ & \\
\hline
\end{tabular}


Héctor Alberto Botello - Andrea López Alba

\begin{tabular}{|c|c|c|c|c|c|c|}
\hline \multirow{2}{*}{ Variable } & \multirow{2}{*}{ Característica } & \multicolumn{4}{|c|}{ Especificación del Modelo } & \multirow{2}{*}{$\begin{array}{c}\text { Característica } \\
\text { Base }\end{array}$} \\
\hline & & (1) & (2) & (3) & (4) & \\
\hline \multirow{7}{*}{$\begin{array}{l}\text { Tamaño } \\
\text { empresa }\end{array}$} & De 2 a 3 personas & $-0,032(0,08)$ & $-0,04(0,05)$ & $-0,0222(0,04)$ & $-0,0497(0,06)$ & Una persona \\
\hline & De 4 a 5 personas & $0,0751(0,02)$ & $0,06(0,05)$ & $0,0687(0,01)$ & $0,0424(0,05)$ & \\
\hline & De 6 a 10 personas & $0,0267(0,02)$ & $0,03(0,04)$ & $0,0166(0,03)$ & $-0,0134(0,03)$ & \\
\hline & De 11 a 20 personas & $0,0993(0,06)$ & $0,09(0,08)$ & $0,0896(0,03)$ & $0,0991(0,1)$ & \\
\hline & De 21 a 50 personas & $0,0155(0,08)$ & $-0,01(0,05)$ & $0,0313(0,09)$ & $-0,0098(0,06)$ & \\
\hline & $\begin{array}{l}\text { De } 51 \text { a } 100 \text { per- } \\
\text { sonas }\end{array}$ & $0,0146(0,04)$ & $0,03(0,05)$ & $0,0123(0,07)$ & $-0,0126(0,09)$ & \\
\hline & $\begin{array}{l}\text { Más de } 100 \text { per- } \\
\text { sonas }\end{array}$ & $0,0723(0,08)$ & $0,05(0,01)$ & $0,0713(0,08)$ & $0,0571(0,09)$ & \\
\hline \multirow{9}{*}{ Rama } & Minería & $-0,3464(0,01)$ & $-0,23(0,07)$ & $-0,3958(0,08)$ & $-0,3536(0,05)$ & Agricultura \\
\hline & Industria & & $0,01(0,04)$ & $-0,0308(0,02)$ & $0,0127(0,09)$ & \\
\hline & Construcción & $0,0698(0,04)$ & $0,09(0,05)$ & $0,069(0,05)$ & $0,1295(0,04)$ & \\
\hline & Utilities & $-0,0154(0,09)$ & $-0,02(0,08)$ & & $0,0396(0,1)$ & \\
\hline & Comercio & $-0,0961(0,07)$ & $-0,09(0,04)$ & $-0,1266(0,05)$ & $-0,0878(0,06)$ & \\
\hline & Transporte & & $-0,02(0,02)$ & $-0,0219(0,03)$ & $-0,0241(0,06)$ & \\
\hline & Salud y educación & $0,079(0,09)$ & $0,08(0,08)$ & $0,0507(0,07)$ & $0,0988(0,07)$ & \\
\hline & $\begin{array}{l}\text { Administración } \\
\text { pública }\end{array}$ & $0,1272(0,06)$ & $0,15(0,01)$ & $0,111(0,01)$ & $0,1782(0,06)$ & \\
\hline & Otros servicios & $-0,0595(0,05)$ & $-0,06(0,02)$ & $-0,0792(0,01)$ & $-0,0336(0,1)$ & \\
\hline Sector & Público & $-0,0677(0,06)$ & $-0,07(0,08)$ & $-0,0739(0,08)$ & $-0,0803(0,01)$ & Privado \\
\hline \multirow{7}{*}{$\begin{array}{l}\text { Lugar de } \\
\text { Trabajo }\end{array}$} & $\begin{array}{l}\text { Su vivienda, sin } \\
\text { instalación }\end{array}$ & $-0,2958(0,02)$ & $-0,28(0)$ & $-0,3063(0,05)$ & $-0,3045(0,03)$ & $\begin{array}{l}\text { Local de la } \\
\text { Empresa }\end{array}$ \\
\hline & $\begin{array}{l}\text { Su vivienda, con } \\
\text { especial }\end{array}$ & $-0,0725(0,03)$ & $-0,06(0,03)$ & $-0,0814(0,08)$ & $-0,0546(0,08)$ & \\
\hline & $\begin{array}{l}\text { Otra vivienda, sin } \\
\text { instalación }\end{array}$ & $-0,2262(0,09)$ & $-0,22(0,06)$ & $-0,2453(0,08)$ & $-0,2404(0,02)$ & \\
\hline & $\begin{array}{l}\text { Otra vivienda, con } \\
\text { instalación }\end{array}$ & $-0,0524(0,07)$ & $-0,05(0,01)$ & $-0,0642(0,01)$ & $-0,0623(0,02)$ & \\
\hline & Puesto ambulante & $-0,0899(0,01)$ & $-0,09(0,05)$ & $-0,0764(0,06)$ & $-0,0995(0)$ & \\
\hline & $\begin{array}{l}\text { Puesto permanente } \\
\text { vía pública }\end{array}$ & $-0,022(0,07)$ & $-0,01(0,01)$ & $-0,0391(0,08)$ & $-0,0432(0,09)$ & \\
\hline & Otro & $0,0286(0,02)$ & $0,04(0,08)$ & $0,0174(0,09)$ & $0,0333(0,1)$ & \\
\hline \multirow{4}{*}{ Estado Civil } & Casada & $-0,069(0,06)$ & $-0,07(0,05)$ & $-0,0702(0)$ & $-0,0878(0,02)$ & Soltera \\
\hline & Divorciada & $-0,2293(0,05)$ & $-0,22(0,07)$ & $-0,2318(0,04)$ & $-0,2432(0,03)$ & \\
\hline & Viuda & $-0,1211(0,03)$ & $-0,12(0,02)$ & $-0,1193(0,1)$ & $-0,1224(0,08)$ & \\
\hline & Unión Libre & $-0,2101(0,07)$ & $-0,22(0,09)$ & $-0,238(0,05)$ & $-0,2226(0,01)$ & \\
\hline $\begin{array}{l}\text { Seguridad } \\
\text { Social }\end{array}$ & Sí & $0,0345(0,03)$ & $0,03(0,02)$ & $0,0314(0,03)$ & $0,0224(0,01)$ & No \\
\hline
\end{tabular}


El efecto de la maternidad sobre los salarios femeninos en Latinoamérica

\begin{tabular}{|c|c|c|c|c|c|c|}
\hline \multirow{2}{*}{ Variable } & \multirow{2}{*}{ Característica } & \multicolumn{4}{|c|}{ Especificación del Modelo } & \multirow{2}{*}{$\begin{array}{c}\text { Característica } \\
\text { Base }\end{array}$} \\
\hline & & (1) & (2) & (3) & (4) & \\
\hline \multirow{10}{*}{$\begin{array}{l}\text { Su nivel } \\
\text { educativo }\end{array}$} & Preescolar & $-0,2824(0,05)$ & $-0,24(0,05)$ & $-0,2816(0,01)$ & $-0,2549(0,02)$ & Ninguno \\
\hline & $\begin{array}{l}\text { Primaria incom- } \\
\text { pleta }\end{array}$ & $-0,0299(0,05)$ & & $-0,015(0,08)$ & $-0,0125(0,02)$ & \\
\hline & Primaria completa & $0,0451(0,08)$ & $0,1(0,05)$ & $0,0584(0,07)$ & $0,0721(0,04)$ & \\
\hline & $\begin{array}{l}\text { Secundaria incom- } \\
\text { pleta }\end{array}$ & $0,0901(0,02)$ & $0,12(0,04)$ & $0,1008(0,07)$ & $0,1191(0,06)$ & \\
\hline & $\begin{array}{l}\text { Secundaria com- } \\
\text { pleta }\end{array}$ & $0,1277(0,09)$ & $0,17(0,03)$ & $0,1255(0,02)$ & $0,1567(0,07)$ & \\
\hline & $\begin{array}{l}\text { Técnica superior } \\
\text { incompleta }\end{array}$ & $0,2648(0,01)$ & $0,29(0,06)$ & $0,2656(0,03)$ & $0,2992(0,08)$ & \\
\hline & $\begin{array}{l}\text { Técnica superior } \\
\text { completa }\end{array}$ & $0,2544(0,03)$ & $0,29(0,06)$ & $0,2597(0,09)$ & $0,3097(0,08)$ & \\
\hline & $\begin{array}{l}\text { Universitaria in- } \\
\text { completa }\end{array}$ & $0,1557(0,09)$ & $0,23(0,01)$ & $0,1757(0,09)$ & $0,1968(0,08)$ & \\
\hline & $\begin{array}{l}\text { Universitaria com- } \\
\text { pleta }\end{array}$ & $0,5196(0,06)$ & $0,56(0,08)$ & $0,5403(0,09)$ & $0,5498(0,02)$ & \\
\hline & Posgrado & $0,6644(0,01)$ & $0,71(0,01)$ & $0,6724(0,02)$ & $0,7293(0,01)$ & \\
\hline Tiene hijos & Sí & $-0,1315(0,04)$ & & & & No tiene \\
\hline \multirow{2}{*}{ Hijos por edad } & $\begin{array}{l}\text { De menos de } 5 \\
\text { años }\end{array}$ & & $-0,2084(0,1)$ & & & No tiene \\
\hline & $\begin{array}{l}\text { De más de cinco } \\
\text { años }\end{array}$ & & & & & \\
\hline $\begin{array}{l}\text { Número de hi- } \\
\text { jos (Continua) }\end{array}$ & & & & $-0,012(0,03)$ & & \\
\hline \multirow{3}{*}{$\begin{array}{l}\text { Hijos por } \\
\text { cantidad }\end{array}$} & Un hijo & & & & $-0,1779(0,06)$ & No tiene \\
\hline & Dos hijos & & & & $\begin{array}{l}-0,06706 \\
(0,02)\end{array}$ & \\
\hline & Tres o más hijos & $0,0174(0,04)$ & $0,0073(0,04)$ & $0,0129(0,07)$ & $0,0144(0,07)$ & \\
\hline \multicolumn{7}{|c|}{ Resultados de la regresión para corregir el sesgo de selección } \\
\hline Casada & No & $0,44(0,06)$ & $0,43(0,05)$ & $0,44(0,06)$ & $0,44(0,06)$ & $\mathrm{Si}$ \\
\hline \multicolumn{2}{|l|}{ Edad } & $0,006(0,002)$ & $0,000(0,002)$ & $0,006(0,002)$ & $0,006(0,00)$ & Continua \\
\hline \multicolumn{2}{|l|}{$\operatorname{Edad}^{\wedge} 2$} & $-0,001(0,00)$ & $-0,002(0,00)$ & $-0,001(0,00)$ & $-0,001(0,00)$ & Continua \\
\hline Área & Urbana & $0,135(0,02)$ & $0,102(0,025)$ & $0,135(0,02)$ & $0,135(0,02)$ & Rural \\
\hline \multicolumn{2}{|l|}{ Constante } & $-2,490105$ & $-2,4715$ & $-2,46538$ & $-2,48712$ & \\
\hline \multirow[t]{2}{*}{$\begin{array}{l}\text { Observaciones } \\
\text { R2 ajustado }\end{array}$} & & 5.215 .419 & 5.215 .419 & 5.215 .419 & 5.215 .419 & \\
\hline & & 0,525 & 0,5334 & 0,5254 & 0,5343 & \\
\hline
\end{tabular}

Todas las variables son significativas al $5 \%$.

Fuente: Cálculos de los autores. Encuesta de la CAF (2012) 
La especificación 3 permite estimar que por cada niño adicional, el salario de la mujer se reduce en un 1,2 \% en promedio. Sin embargo, cuando se distribuye este efecto por la cantidad de niños en el hogar (especificación 4), se aprecia que si la mujer tiene un hijo, el salario promedio disminuye en un 17,8 \%; si tiene dos hijos, el $6 \%$, y si tiene más de dos hijos, la baja es de un $12 \%$. Este resultado, primero un diferencial creciente y luego decreciente, indicaría que en términos econométricos la constitución de la especificación 3 no sería lineal sino cuadrática y sería necesario replantear el modelo para mejorar la interpretación del mismo.

Sobre las estimaciones se obtuvieron otros resultados de interés:

- La educación es la mejor inversión que pueden hacer las mujeres para incrementar su ingreso laboral por hora. En este orden de ideas, una mujer con grado universitario gana en promedio un $58 \%$ más que una mujer que no tiene ningún grado educativo; si tiene técnico es un $41 \%$; secundaria, un $25 \%$, y primaria, un $15 \%$.

- Las madres solteras llegan a ganar entre un $7 \%$ y un $8 \%$ menos que las mujeres que trabajan y están con su pareja en el hogar. Esta tendencia se mantiene con otros tipos de estados civiles.

- El ocupar cargos en grandes empresas brinda la posibilidad de mejores remuneraciones, entre un $7 \%$ y un $8 \%$ más altos que las mujeres que trabajan por su cuenta. Igualmente las empresas que contribuyen con seguridad social hacen que las mujeres ganen un 3 \% más. En relación con su lugar de trabajo, las formas informales tales como trabajar en la vivienda, o en puestos de ventas en las vías públicas tienen una fuerte penalización, 10 \% por debajo de la media.

- Los sectores de la construcción y servicios ofrecen a las mujeres los mejores ingresos laborales, con salarios entre un $7 \%$ y un $9 \%$ por encima de la media. Las mujeres que trabajan en el sector público ganan menos que en el sector privado, aproximadamente un $7 \%$.

\section{Descomposición de Oaxaca Blinder}

La tabla 7 muestra los resultados de la descomposición salarial a través de la metodología Oaxaca Blinder. Primero se obtienen los valores esperados de la variable dependiente (el logaritmo del salario) para cada grupo. Luego se estima el diferencial entre los mismos. A continuación, se separan los efectos provenientes de los coeficientes y de la variable de control. Todos estos procesos se realizan por modelos, uno que no realiza la corrección del sesgo de selección y otro que sí lo corrige, tal y como se expuso en la parte metodológica. 
El efecto de la maternidad sobre los salarios femeninos en Latinoamérica

Tabla 7. Resultados de la descomposición Oaxaca Blinder.

Errores estándar entre paréntesis

\begin{tabular}{|l|c|c|}
\hline \multicolumn{1}{|c|}{$\begin{array}{c}\text { Grupo de predicción } \\
\text { Ingreso laboral por hora (en dólares corrientes) }\end{array}$} & Sin corrección & Corregido \\
\hline No Madres & $6,41(0,001)$ & $6,39(0,086)$ \\
\hline Madres & $6,28(0,001)$ & $6,23(0,065)$ \\
\hline Diferencia & $-0,13(0,001)$ & $-0,16(0,108)$ \\
\hline Explicada & $0,1(0,001)$ & $0,09(0,071)$ \\
\hline No explicada & $\begin{array}{c}-0,23 \\
(0,001)\end{array}$ & $-0,27(0,091)$ \\
\hline
\end{tabular}

Fuente: Cálculos de los autores. Encuesta de la CAF (2012)

Se observa que sin la corrección, la brecha por maternidad es del $13 \%$, mientras que con la corrección es del $16 \%$. En este orden de ideas, la dotación entre los grupos explica cerca de dos terceras partes de diferencial, con el $10 \%$ y el $9 \%$, respectivamente. Mientras que el porcentaje restante (-23\% y - 27\%) está explicado por variables no observables incluyendo la condición de maternidad. Es decir, que si se seleccionaran aleatoriamente a una mujer madre y a otra que no lo es, y se les asignasen los mismos niveles de productividad, dotación de capital humano, experiencia y puesto de trabajo, la madre tendría un ingreso laboral por hora $16 \%$ menor, explicado por la discriminación por parte de los empleadores en el momento de la contratación.

\section{CONCLUSIONES}

La penalización por maternidad en la muestra de nueve países de América Latina que surge después de controlar por variables observables como la educación, la estructura del hogar y las características laborales es de alrededor de $13 \%$; sin embargo, esta brecha está localizada en los rangos más altos de educación. Esta resulta ser mayor cuando la madre tiene niños de menos de cinco años, con el 21 \%.

Con la ayuda de la descomposición Oaxaca Blinder, se obtuvo que la brecha se encuentra explicada mayormente por la condición de maternidad y la discriminación institucional por parte de los empleadores. Los resultados también confirman que las madres tienen una mayor tendencia a estar ubicadas en empleos informales de baja productividad y, en consecuencia, con bajos salarios.

Con base en lo anterior, si bien las políticas existentes como las licencias de maternidad remuneradas y la prohibición del despido a las embarazadas evitan las 
interrupciones de carrera y el flujo de ingresos en el corto plazo, las políticas orientadas a la disminución de la brecha por maternidad deben lograr que las madres tengan menores pérdidas en términos de experiencia laboral y educación en un horizonte más largo del tiempo especialmente para mujeres con hijos en edades inferiores a los cinco años.

En este sentido deben diseñarse políticas que aminoren los desincentivos potenciales de contratar una mujer, tales como la menor dedicación laboral como consecuencia del cuidado de los hijos. Además de los costos adicionales que conlleva la empresa de contratar empleados para cubrir la vacante que deja la empleada al entrar en una licencia de maternidad.

Finalmente este artículo presenta limitaciones. La exploración de diferentes países presenta un problema de contextualización espacial de la situación de las mujeres. No se pudieron controlar características no observables como la motivación, el compromiso con el trabajo y otros factores asociados a las características de la firma. Tampoco se puede observar la evolución de las diferencias salariales a medida que el mismo individuo pierde o adquiere características de manera inter-temporal; se podrían utilizar datos de manera longitudinal que permitan el seguimiento de los trabajadores.

\section{BIBLIOGRAFÍA}

Albrecht, James; Per-Anders, Edin; Marianne, Sundström y Susan B. Vroman (1999). Career interruptions and subsequent earnings: a reexamination using Swedish data. En:Journal of Human Resources, Vol. 34, No. 2, p. 294-311.

Asplund, Rita, y Pedro Telhado Pereira (1999). Returns to Human Capital in Europe. A Literature Review, [En línea] The Research Institute of the Finnish Economy, Helsinki, 368p. [Consultado el 2 de diciembre de 2013].

Atucha, Ana y Actis, Pasquale. (2003). Brechas salariales: discriminación o diferencias de productividad. En: Revista Momento Económico, Vol. 126, p. 23-33.

Baxter, Janeen. (1992). Domestic Labour and Income Inequality. En: Employment and Society, Vol. 6, No. 2, p. 229-249.

Becker, Gary (1985). Human capital effort, and the sexual division of labour. En: Journal of Labour Economics, Vol. 3, No. 1, Part 2, p. 33-58.

Budig, Michelle y Paula England. (2001).The Wage Penalty for Motherhood. En: American Sociological Review, 66, Vol. 66, No. 2, p 204-225. 
Ben-Porath, Yoram. (1967). The production of human capital and the life cycle of earnings. En: The Journal of Political Economy, Vol. 75, No. 4, p. 352-365.

CAF-Corporación Andina de Fomento-(2012). Encuesta CAF. Corporación Andina de Fomento y Banco de Desarrollo de América Latina.

Cárdenas, Mauricio y Rozo, Sandra. (2009). Informalidad empresarial en Colombia: problemas y soluciones. Desarrollo y Sociedad, Vol. 63, No. 1, p. 211-243.

Daymont, Thomas y Andrisani, Paul (1984). Job preferences, college major, and the gender gap in earnings. En: Journal of Human Resources, Vol. 19, No. 3, p. 408-428.

Esquivel, Valeria (2007). Género y Diferenciales de Salarios en la Argentina. En: M. Novick and H. Palomino, edit. Estructura productiva y empleo: un enfoque transversal. Buenos Aires, Argentina: Ministerio de Trabajo, Empleo y Seguridad Social, p. 363-392.

Fuentes, Jeanette; Palma, Amalia y Montero, Rodrigo (2005). Discriminación salarial por género en Chile: una mirada global. En Estudios de Economía, Vol. 32, No. 2, p. 133-157.

Gupta, Datta y Smith, Nina (2002). Children and career interruptions: the family gap in Denmark. En: Económica, Vol. 69, No. 276, p. 609-629.

Gutiérrez, Diego (2008). Efectos de la fecundidad sobre el ingreso laboral femenino. Bogotá, 40 p. Tesis (Magíster en Economía). Universidad de Los Andes. Facultad de Economía.

Harmon, Colm; Westergaard-Nielsen y Ian Walker (Eds.) (2001). Education and earnings in Europe: a cross-country analysis of the returns to education. Edward Elgar Publishing, 332p.

Harmon Colm, Hessel Oosterbeek, y Ian Walker (2003). The Returns to Education: Microeconomics, En Journal of Economic Surveys, Vol. 17 No. 2, p. 115-155.

Harkness, Susan, y Jane Waldfogel (2003). The family gap in pay: evidence from seven industrialized countries. En: Research in Labor Economics, Vol 22, No.1, p.369-414.

Heckman, James (1979). Sample selection bias as a specification error. En: Econométrica, Vol. 47, No. 1, p.153-161.

Hyclak, Thomas. (2012). Fundamentals of labor economics. Cengage Learning.528p.

Jacobsen, Joyce y Levin, Laurence. (1995). Effects of intermittent labor force attachment on women's earnings. En: Monthly Labor Review, Vol. 118, No.9, p. 14-19.

Joshi, Heather y Newell, Marie (1989). Pay Differentials and Parenthood: Analysis of Men and Women Born in 1946. University of Warwick Institute for Employment Research. 104p.

Joshi, Heather, Paci Pierella, y Waldfogel Jane (1999). The wages of motherhood: better or worse? En: Cambridge Journal of Economics, Vol. 23, No. 5, pp. 543-564. 
Korenman, S., y Neumark, D. (1992). Marriage, motherhood, and wages. En: Journal of Human Resources, Vol. 27, No. 2, p. 233-255.

Livermore, Tanya, Joan Rodgers, y Peter Siminski (2011). The Effect of Motherhood on Wages and Wage Growth: Evidence for Australia. En: Economic Record, Vol. 87 No. 1, p. 80-91.

Martínez Jasso y Acevedo Gloria (2004). La brecha salarial en México con enfoque de género: capital humano, discriminación y selección muestral. En: Ciencia Uanl, Vol. 7, No.1, p. 66-71.

Mincer, Jacob (1974). Schooling, Experience and Earnings. National Bureau of Economic Research. 187p.

Molina, José y Montuenga Víctor (2008). The Motherhood Wage Penality in a Mediterranean Country: The Case of Spain. En: IZA Discussion Papers 3574, Institute for the Study of Labor, Bonn (Germany).

Olarte, Liliana y Peña Ximena. (2010). El efecto de la maternidad sobre los salarios femeninos. En: Documento CEDE, (2010-18).

Oaxaca, Ronald (1973). Male-female wage differentials in urban labor markets. En International economic review, Vol. 14, No.3, p. 693-709.

Peñas, Ignacio (2002). La discriminación salarial por razones de género: un análisis empírico del sector privado en España. En Reis, Vol. 98, No. 2, p. 171-196.

Psacharopoulos, Gary y Patrinos Harry (2004). Returns to investment in education: a further update. En: Education economics, Vol. 12, No. 2, p. 111-134.

Rivera, Jairo (2013). Teoría y práctica de la discriminación en el mercado laboral ecuatoriano (2007-2012). En: Analítika, Vol. 5, No.1, p. 3-18.

Rodríguez, José e Higa Minoru (2009). Informalidad, empleo y productividad en el Perú. En: Documentos de Economía, Vol. 28, No. 1, p. 1-15.

Rosen Sherwin (1986). The theory of equalizing differences. En: Handbook of labor economics, Cap 12, p. 641-692.

Samaniego, Norma (2009). El crecimiento explosivo de la economía informal. En Economía, UNAM, Vol. 5, No.13, p. 30-41.

Todd, Erin (2001). Educational attainment and family gaps in women's wages: evidence from five industrialized countries. Luxembourg Income Study, LIS WP. N. 246p.

Torres, Víctor y Celton Dora (2009). Discriminación salarial en Argentina entre nativos y paraguayos. En Cuadernos Geográficos de la Universidad de Granada, Vol. 45, No. 2, p. 263-285. 
Waldfogel, Jane. (1995). The price of motherhood: family status and women's pay in a young British cohort. En:Oxford Economic Papers, Vol. 47, No.4, p. 584-610.

Waldfogel, Jane. (1997). The effect of children on women's wages. En: American sociological review, Vol. 62, No.42, p. 209-217. 
